Check for updates

Cite this: RSC Adv., 2019, 9, 39595

Received 10th July 2019

Accepted 29th October 2019

DOI: $10.1039 / c 9 r a 05280 a$

rsc.li/rsc-advances

\section{Influence of uniform magnetic field on physicochemical properties of freeze-thawed avocado puree}

\author{
Yinying Tan, ${ }^{\text {ab }}$ Yamei Jin, ${ }^{\text {ab }} \mathrm{Na}$ Yang, (D) ${ }^{\mathrm{b}}$ Zhe Wang, ${ }^{\mathrm{b}}$ Zhengjun Xie, (D) *ab

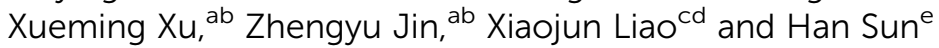

\begin{abstract}
External fields are reported to have the potential to improve the quality of food after freeze-thawing. This study investigated the effect of an oscillating magnetic field with high uniformity and multi-directions at 4 $\mathrm{mT}$ and $50 \mathrm{~Hz}$ on the freezing of avocado puree. Samples of avocado puree were placed in the central zone of three pairs of orthometric Helmholtz coils where a highly homogeneous degree ( $99 \%$ homogeneity) magnetic field could be guaranteed. For comparison, avocado puree was frozen without the magnetic field. According to the freezing curve, under the magnetic field, the freezing point of avocado puree was decreased from -1.2 to $-6{ }^{\circ} \mathrm{C}$, which indicated that the temperature zone for maximum ice crystal formation was reduced. The uniform magnetic field had a positive effect on the avocado puree. The $\mathrm{pH}$ value of the samples after the magnetic field-assisted freezing was higher than that of the control. With the increase in the magnetic field direction, $\Delta E$ gradually decreased. In addition, the antioxidant activity of the puree significantly decreased, and the diphenylpicrylhydrazyl (DPPH) radical scavenging activity decreased from $69.18 \%$ to $56.22 \%$, whereas the ferric reducing antioxidant power (FRAP) value decreased from $18.5 \%$ to $14.75 \%$. However, the three-direction magnetic field-assisted freezing could better maintain the antioxidant activities of avocado puree. Moreover, the interaction of ferrous gluconate and multi-directional magnetic field could exert an improvement on the quality of freezethawed avocado puree, owing to the small molecular current.
\end{abstract}

\section{Introduction}

Avocado is popular with consumers worldwide, and is rich in protein, minerals, vitamins, and bioactive compounds. Avocado is also a high-energy, low-sugar fruit (only about $0.2 \mathrm{~g}$ sugar per one-half fruit), which confirms with the healthy food requirements for humans. ${ }^{1}$ Avocado puree is a typical frozen food often served with corn chips as an appetizer in restaurants. During preservation and transportation, freezing not only extends the shelf life of the products but also helps maintain their sensory quality.

Numerous studies have reported that applying external fields during the freezing process can reduce the size of ice crystals to protect the cells or tissues from freezing damage. Thus, food

${ }^{a}$ State Key Laboratory of Food Science and Technology, Jiangnan University, 1800 Lihu Road, Wuxi 214122, PR China. E-mail: xiezj@jiangnan.edu.cn; Fax: + 86510 85913612; Tel: + 8651085913612

${ }^{b}$ School of Food Science and Technology, Jiangnan University, 1800 Lihu Road, Wuxi 214122, PR China

${ }^{c}$ College of Food Science and Nutritional Engineering, China Agricultural University, Beijing 100083, China

${ }^{d}$ Beijing Key Laboratory for Food Non-Thermal Processing, Beijing 100083, China

${ }^{e}$ INDUC Scientific Co., Ltd., No. 28-132 Jinshan North Photoelectric Science and Technology Park, Wuxi 214000, China qualities are better maintained, to some extent, after thawing. Recently, various non-conventional freezing techniques in the food industry, in which electric field, ${ }^{2,3}$ magnetic field, ${ }^{4}$ electromagnetic wave, ${ }^{5,6}$ ultrasonic wave, ${ }^{7,8}$ as well as pulsed electric field plus oscillating magnetic field are used as stimulus, have been investigated to improve the quality of frozen food. ${ }^{9}$

Dalvi-Isfahan et al. applied an electrostatic field (ESF) during the freezing of lamb meat, which reduced the drip loss after thawing and helped maintain the hardness and microstructure of the meat sample. Meanwhile, no color decline was observed after ESF freezing. ${ }^{10}$ Sun et al. investigated the relationship between ice crystal formation rate and electric field frequency at 1-200 kHz. They found that the effectiveness of the field was frequency-dependent, with an increase in frequency, both the size of ice crystals and the freezing time were decreased, and the smallest ones were obtained at $50 \mathrm{kHz} .^{11}$ Pulsed electric field combined with static magnetic field exerted a significant effect on the freezing of electrolyte solution, thus the solution had a tendency to form tiny ice crystals. ${ }^{12}$ Also, appropriate microwave radiation at low power level during the freezing was found to decrease the average size of ice crystals in meat tissue. ${ }^{5}$ Cheng et al. demonstrated that the application of an ultrasound field at a specific temperature during the freezing process enabled ice nucleation at a low degree of super-cooling. ${ }^{13}$ Zhao et al. 
claimed that the preservation quality of cucumbers could be improved by using the static magnetic field combined with cold water shock treatment $\left(B=70 \mathrm{Gs}, T_{\mathrm{w}}=2{ }^{\circ} \mathrm{C}\right) .{ }^{14}$ However, some investigations on magnetic field-assisted freezing of foods found that oscillating magnetic fields $(<3 \mathrm{mT})$ had no effect on the improvement of quality factors. ${ }^{15}$

Although many investigations have been conducted on the effect of the magnetic field on food quality, it remains largely controversial. This is due to the extremely-low magnetic conductivity (relative magnetic permeability, $u_{\mathrm{r}} \approx 1$ ) of food materials, which leads to the effect of magnetic field-assisted freezing on food that is too weak to be detected. Furthermore, some factors such as the uniformity and the direction of magnetic field are usually neglected in the analysis, which contributes to the inconsistent experimental findings. In this study, an oscillating magnetic field with high uniformity and different direction (one-direction, 1D; two-direction, 2D; threedirection, 3D) was applied to freeze avocado puree (Persea americana Mill.). The influence of the magnetic field on physicochemical properties of avocado puree after freeze-thawing, including freezing parameters, $\mathrm{pH}$ value, moisture content, soluble solids content, antioxidant activity, and color difference were analyzed. In contrast to whole pieces of fruits, the pureed food is more amenable to the addition of nutrient supplements (ferrous ions) to enhance the effect of magnetic field during the freezing process. Additionally, the relative magnetic permeability of avocado puree was determined, and the combined effect of ferrous ions and magnetic field on the freezing were thus evaluated.

\section{Materials and methods}

\subsection{Materials and reagents}

Fresh 'Hass' avocados (Persea americana Mill.) at the same ripening stage were purchased from a supermarket in Wuxi, Jiangsu, China. After peeling, the avocados were cut into cubes of $0.5 \mathrm{~cm} \times 0.5 \mathrm{~cm} \times 0.5 \mathrm{~cm}$ and blended into puree. In order to investigate the effect of Fe fortifier on the uniform magnetic field-assisted freezing of avocado puree, ferrous gluconate was also added during the blending process, and evenly mixed (ensuring $15 \mathrm{mg} \mathrm{Fe} \mathrm{kg}{ }^{-1}$ diet). ${ }^{\mathbf{1 6}}$ All analytical-grade chemicals and solvents were supplied by Sinopharm Group Co., Ltd (Shanghai, China).

\subsection{Experimental system}

An experimental batch air-blast freezer (JD-AC4, INDUC Scientific Co., Ltd., Wuxi, China) which included a magnetic-field generator, fan, control panel, freezing chamber, and refrigeration unit, was used for the freezing. The magnetic-field generator consisted of three pairs of Helmholtz coils $(80 \mathrm{~cm} \times 80 \mathrm{~cm}$ square; 400 turns) and a power supply, which generated a uniform magnetic field at the excitation current of $8 \mathrm{~A}$ and frequency of $50 \mathrm{~Hz}$. The sample chamber dimensions were $20 \mathrm{~cm} \times 20 \mathrm{~cm} \times 20 \mathrm{~cm}$ (Fig. 1a). The cooling temperature range was from -20 to $10{ }^{\circ} \mathrm{C}$. A BLD-1030 Gaussian meter (Bolandun Corporation Ltd., Beijing, China) and a space microdisplacement platform were used to measure the field strength and its homogeneity in the chamber. At these settings, the maximum measured value was $4 \mathrm{mT}$ (AC), and the homogeneity of the magnetic field was 99\%. Moreover, numerical simulation of the magnetic field in freezing chamber was performed using the COMSOL multiphysics $4.3 \mathrm{~b}$ software (Fig. 1b).

\subsection{Freeze-thaw procedure}

The experiments were performed using the above-mentioned system. The magnetic field parameters are listed in Table 1. The cooling temperature was kept at $-20{ }^{\circ} \mathrm{C}$. The puree was added into $25 \mathrm{~mL}$ beakers (50 g puree, $35 \mathrm{~mm}$ diameter), covered with plastic film and placed in the chamber. The samples were frozen under the magnetic field until their temperature reached $-18{ }^{\circ} \mathrm{C}$ (about $4 \mathrm{~h}$ ). After freezing, the samples were thawed at $10{ }^{\circ} \mathrm{C}$, until their temperature reached $8{ }^{\circ} \mathrm{C}$ (about $10 \mathrm{~h}$ ). Then the physicochemical properties of the samples were immediately analyzed.

\subsection{History of temperature}

During processing, Type $\mathrm{T}$ (copper/constantan) thermocouple wires ( $1 \mathrm{~mm}$ diameter) connected to a data logger (Eltek Type 1000, Grant Instruments Co., Ltd. Cambridge, UK) were located at the geometric center of the beakers filled with avocado puree

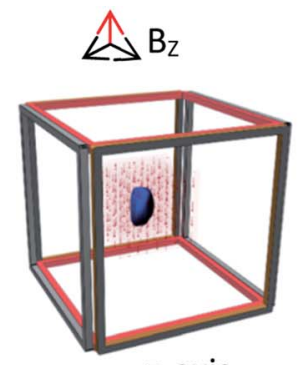

z-axis

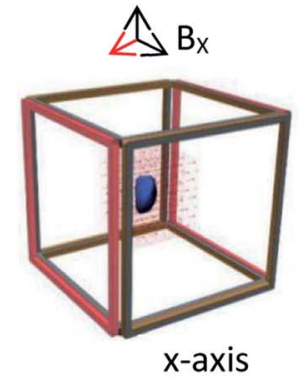

$\mathrm{x}$-axis

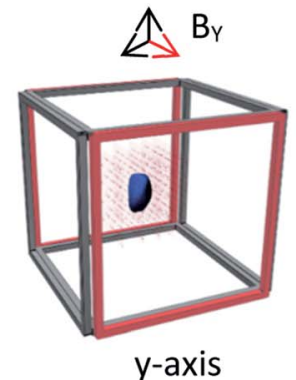

y-axis

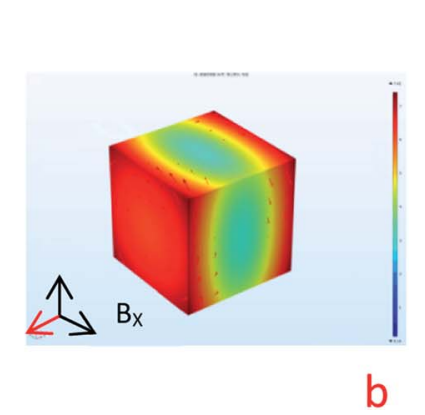

Fig. 1 Freeing under uniform magnetic field at $50 \mathrm{~Hz}$ in different directions. (a) Orthometric Helmholtz coils excited in different directions for interval. (b) Numerical simulation of the one-direction magnetic field $\left(B_{X}\right)$ in freezing chamber (maximum magnetic field strength, 4.2 mT; uniformity, 99.9\%; excitation current, 8 A). 
Table 1 Parameters of the oscillating magnetic fields

\begin{tabular}{|c|c|c|c|c|c|c|}
\hline Applied magnetic fields & Direction & Magnetic field intensity/mT & Interval time $/ \mathrm{min}$ & Frequency/Hz & $\begin{array}{l}\text { Homogeneity } \\
\text { degree }\end{array}$ & Periodic time $/ \mathrm{min}$ \\
\hline$B_{X}$ (one-direction, 1D) & $x$-axis & 4 & 2 & 50 & $98 \%$ & - \\
\hline \multirow[t]{2}{*}{$B_{X+Y}($ two-direction, 2D) } & $x$-axis & 4 & 2 & 50 & $98 \%$ & 4 \\
\hline & $y$-axis & 4 & 2 & 50 & $98 \%$ & \\
\hline \multirow[t]{3}{*}{$B_{X+Y+Z}$ (three-direction, 3D) } & $x$-axis & 4 & 2 & 50 & $98 \%$ & 6 \\
\hline & $y$-axis & 4 & 2 & 50 & $98 \%$ & \\
\hline & $z$-axis & 4 & 2 & 50 & $98 \%$ & \\
\hline
\end{tabular}

samples. Then, the temperature of the samples could be recorded.

\subsection{Relative magnetic permeability}

The relative magnetic permeability of the sample was determined using the 65120B Impedance Analyzer (Wayne Kerr Electronic. West Sussex, UK) with a 1030 relative magnetic permeability probe installed on the $1101 \mathrm{~J}$ fixture component. The probe was calibrated by testing the resistance $\left(R_{\mathrm{W}}\right)$ and inductance $\left(L_{\mathrm{w}}\right)$ of an air coil (number of winding, $N=1$ ) wound around the magnetic circuit. The calibration parameters were $L$ $=64.6 \mathrm{~mm}$ (length of the magnetic circuit) and $A=45 \mathrm{~mm}^{2}$ (cross-sectional area of the magnetic circuit). After calibration, the sample was placed in the air coil groove (center circumference, $64.6 \mathrm{~mm}$; cross-sectional area, $45 \mathrm{~mm}^{2}$ ) of the probe. After tightening the upper cover, the relative magnetic permeability was recorded (Fig. 2).

\section{6 pH value, moisture, ferrous, and soluble solids content}

$\mathrm{pH}$ meter and digital refractometer were used to analyze the $\mathrm{pH}$ and soluble solid content (SSC) of the sample. Ferrous and moisture contents were determined in accordance with AOAC $2000 .{ }^{17}$

\subsection{Color assessment}

The samples were evenly coated in a dish for color detection with the USPRO Colorimeter (UltraScan Pro1166, USA). The method was that recommended by the International Commission on Illumination or CIE. And the color difference $(\Delta E)$ was expressed using $L, a, b$ values of treated sample, as follows:

$$
\Delta E=\sqrt{\left(L^{*}-L_{0}^{*}\right)^{2}+\left(a^{*}-a_{0}^{*}\right)^{2}+\left(b^{*}-b_{0}^{*}\right)^{2}}
$$

where $L_{0}^{*}, a_{0}^{*}, b_{0}^{*}$, are the values of fresh avocado puree.

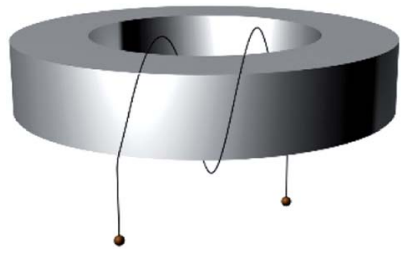

Fig. 2 Measurement of relative magnetic permeability of paste food.

\subsection{Antioxidant capacity}

In present study, the antioxidant activity was evaluated by DPPH and FRAP assay. ${ }^{18,19}$ Avocado puree of $15 \mathrm{~g}$ was mixed with a $10 \mathrm{~mL} 80 \%$ methanol solution and homogenized. The antioxidant activity of the supernatant was measured after centrifugation at $6500 \mathrm{~g}$ and $4{ }^{\circ} \mathrm{C}$ for $20 \mathrm{~min}$.

2.8.1 DPPH* assay. A $200 \mu \mathrm{L}$ aliquot of the supernatant solution was added into a $3.8 \mathrm{~mL}$ volume of freshly prepared DPPH solution $\left(60 \mu \mathrm{mol} \mathrm{L}{ }^{-1}\right.$, dissolved in $\left.\mathrm{HCl}\right)$. The mixture was kept in the dark for $60 \mathrm{~min}$, and subsequently the absorbance was measured at $517 \mathrm{~nm}$ with a spectrophotometer. The DPPH radical scavenging rate was calculated as follows:

$$
\text { DPPH radical scavenging } \operatorname{rate}(\%)=\frac{A_{1}-\left(A_{2}-A_{0}\right)}{A_{1}} \times 100 \%
$$

where $A_{1}, A_{2}$, and $A_{0}$ denote the absorbance levels of the control, the sample, and the blank, respectively.

2.8.2 FRAP assay. The FRAP reagent consisted of $25 \mathrm{~mL}$ of sodium acetate buffer solution ( $\left.0.3 \mathrm{~mol} \mathrm{~L}^{-1}, \mathrm{pH} 3.6\right), 2.5 \mathrm{~mL}$ of $10 \mathrm{mmol} \mathrm{L}{ }^{-1}$ TPTZ solution, and $2.5 \mathrm{~mL}$ of $20 \mathrm{mmol} \mathrm{L}^{-1}$ $\mathrm{FeCl}_{3} \cdot 6 \mathrm{H}_{2} \mathrm{O}$ solution with $2.7 \mathrm{~mL}$ FRAP reagent. $90 \mathrm{~mL}$ supernatant and $270 \mathrm{~mL}$ deionized water were mixed and then reacted at room temperature $\left(25^{\circ} \mathrm{C}\right)$ in the dark for $30 \mathrm{~min}$. The ferric reducing ability of avocado puree was measured by monitoring the increase in absorbance at $593 \mathrm{~nm}$. With methanol instead of the sample as the blank sample and Trolox as a reference, then the standard curve was plotted. The result was expressed as of $\mu \mathrm{mol}$ Trolox equivalent per gram of puree $(\mu \mathrm{mol}$ TE $\mathrm{g}^{-1} \mathrm{DW}$ ).

\subsection{Statistical analysis}

All the experiments were performed in triplicate. The SPSS version 16.0 software was used to analyze the data. Significant differences in the treatments were evaluated by one-way analysis of variance (ANOVA; 95\% significance level) and Duncan's test. All experimental data are expressed as the mean \pm standard deviation (SD).

\section{Results}

\subsection{Temperature history and freezing characteristics}

The freezing point of avocado puree significantly decreased under the uniform magnetic field, and the temperature of the samples were lower than that of the control during freezing (Fig. 3 and 


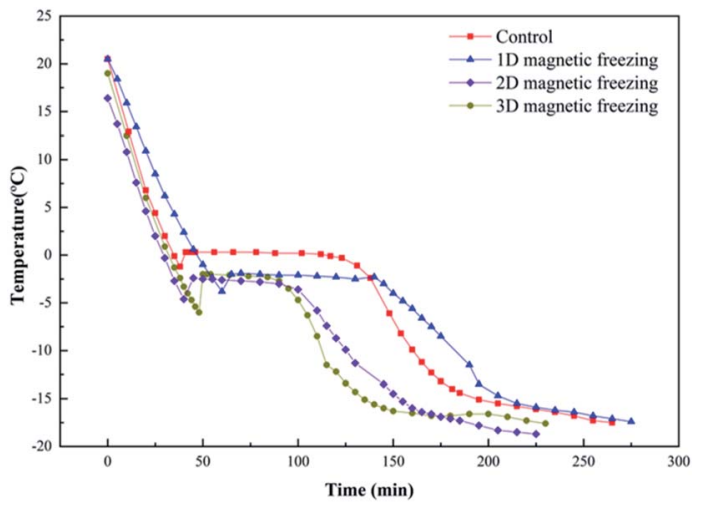

Fig. 3 Temperature history of avocado puree during the freezing with or without uniform magnetic field.

Table 2). The temperature zone for maximum ice crystal formation also showed a significant downward trend. The phase transformation time of avocado puree was respectively shortened by $38.09 \%$ (under $B_{X}$ ), $33.33 \%$ (under $B_{X+Y}$ ), $40.95 \%$ (under $B_{X+Y+Z}$ ) during the process. The faster the sample temperature went through the temperature zone of maximum ice crystal formation, the higher the degree of supercooling. In this case, tiny and uniform ice crystals were formed within the tissues. ${ }^{20}$

Ice crystal formation in biological aqueous solutions during freezing under pulsed magnetic fields (at $6.5 \mathrm{mT}$ ) was investigated. The results revealed that tiny ice crystals formed in the early phase of super-cooled state. By applying alternating magnetic fields, the gap zones created by ice crystal particles after thawing in sweet potato tissues were considerably smaller than quick-freezing process. ${ }^{\mathbf{2 1}, 22}$ When freezing carp and potatoes under static magnetic fields, the phase transformation process was significantly influenced, leading to the shortening of the phase-change time of the sample by more than $20 \%$, relative to that of the control. ${ }^{23}$

\section{2 $\mathrm{pH}$ value, soluble solids content and moisture content}

The $\mathrm{pH}$ values of the samples after uniform magnetic fieldassisted freezing were higher than that of the control. The influence of ice crystals on the non-freezing phase led to the dissolution of soluble organic acids, thereby changing the ionic concentration and $\mathrm{pH}$ of the puree. It implied that tiny ice crystal particles had appeared and less soluble organic acids were dissolved under the uniform magnetic field. As previously reported, the $\mathrm{pH}$ value $(\mathrm{pH}=3.39)$ of strawberry treated with alternating magnetic fields $\left(4.22 \mathrm{~A} \mathrm{~m}^{-1}\right)$ during freezing was lower than that of the control $(\mathrm{pH}=3.62)$ due to the reduced consumption of organic acids during frozen storage. ${ }^{24}$

The soluble solids content in frozen avocado puree was generally higher than that in fresh samples, which was attributed to ice crystals formation. Besides degrading the quality of the puree to some extent, it would induce the dissolution of certain compounds, particularly polysaccharides, resulting in an increase in soluble solids. According to the above findings, the uniform magnetic field exerted a protective effect on the avocado puree, and the effect of 3D magnetic field was better than that of the $1 \mathrm{D}$ field.

The groups arranged in an increasing order according to their moisture content were as follows: 3D magnetic field, fresh puree, 1D magnetic field, 2D magnetic field, and the control (Table 3). These results suggested that the freezing maintained the moisture content of avocado puree under uniform magnetic field. Thus, the loss rate of the moisture content was decreased. As previously reported, the packaged pork, frozen with the assistance of a moderate electromagnetic wave at $-55{ }^{\circ} \mathrm{C}$ and thawed at room temperature, showed a comparatively high moisture content $(62.1 \% \pm 0.5)$ than the samples frozen by air blast $(61.5 \% \pm 0.5) .{ }^{25}$ The result is also consistent with the finding of Kim et al. that an electro-magnetic field usually has a positive effect on the quality of frozen beef. ${ }^{26}$

\subsection{Color assessment}

As shown in Table 4 , the $\Delta E$ in the $\left(B_{X+Y+Z}\right)$ magnetic field group was lower than that of the control. With an increase in the

Table $3 \mathrm{pH}$, SSC and moisture content of avocado puree after the freezing with or without uniform magnetic field ${ }^{a}$

\begin{tabular}{llll}
\hline Samples & $\mathrm{pH}$ & $\mathrm{SSC}$ & Moisture content \\
\hline Fresh puree & $6.50 \pm 0.02^{\mathrm{a}}$ & $0.9 \pm 0.1^{\mathrm{b}}$ & $73.76 \% \pm 0.26 \%^{\mathrm{a}}$ \\
Control & $6.01 \pm 0.03^{\mathrm{a}}$ & $2.5 \pm 0.3^{\mathrm{a}}$ & $71.68 \% \pm 0.33 \%^{\mathrm{d}}$ \\
Freezing under $B_{X}$ & $6.23 \pm 0.01^{\mathrm{a}}$ & $2.2 \pm 0.3^{\mathrm{a}}$ & $72.70 \% \pm 0.19 \%^{\mathrm{b}}$ \\
Freezing under $B_{X+Y}$ & $6.22 \pm 0.02^{\mathrm{a}}$ & $2.1 \pm 0.2^{\mathrm{a}}$ & $72.23 \% \pm 0.17 \%^{\mathrm{c}}$ \\
Freezing under $B_{X+Y+Z}$ & $6.20 \pm 0.05^{\mathrm{a}}$ & $2.1 \pm 0.1^{\mathrm{a}}$ & $73.98 \% \pm 0.22 \%^{\mathrm{a}}$
\end{tabular}

${ }^{a}$ All data are mean \pm SD. Different superscripted letters indicate significant difference within the same volume $(P<0.05)$.

Table 2 Freezing characteristics of avocado puree with or without uniform magnetic field ${ }^{a}$

\begin{tabular}{|c|c|c|c|c|}
\hline Parameters & Control & Under magnetic field $\left(B_{X}\right)$ & Under magnetic field $\left(B_{X+Y}\right)$ & $\begin{array}{l}\text { Under magnetic field } \\
\left(B_{X+Y+Z}\right)\end{array}$ \\
\hline Freezing point $\left({ }^{\circ} \mathrm{C}\right)$ & $-1.2 \pm 0.2$ & $-3.8 \pm 0.3$ & $-4.6 \pm 0.2$ & $-6.0 \pm 0.4$ \\
\hline Phase transition time (min) & $105 \pm 5$ & $85 \pm 2$ & $65 \pm 4$ & $62 \pm 2$ \\
\hline
\end{tabular}

${ }^{a}$ All data are mean \pm SD. Different superscripted letters represent significant difference within the same volume $(P<0.05)$. 
Table 4 Color characteristics of fresh avocado puree after freezing with or without uniform magnetic field ${ }^{a}$

\begin{tabular}{lccc}
\hline Samples & $L$ & $a$ & $b$ \\
\hline Fresh puree & $54.67 \pm 2.82^{\mathrm{b}}$ & $-5.10 \pm 0.49^{\mathrm{b}}$ & $30.83 \pm 1.27^{\mathrm{b}}$ \\
Control & $60.07 \pm 2.53^{\mathrm{a}}$ & $-8.62 \pm 0.89^{\mathrm{c}}$ & $30.38 \pm 2.05^{\mathrm{b}}$ \\
Freezing under $B_{X}$ & $60.29 \pm 1.39^{\mathrm{a}}$ & $-8.69 \pm 0.25^{\mathrm{c}}$ & $36.30 \pm 1.58^{\mathrm{a}}$ \\
Freezing under $B_{X+Y}$ & $55.40 \pm 1.87^{\mathrm{b}}$ & $-4.98 \pm 0.02^{\mathrm{b}}$ & $31.64 \pm 1.38^{\mathrm{b}}$ \\
Freezing under $B_{X+Y+Z}$ & $46.17 \pm 2.37^{\mathrm{c}}$ & $-3.22 \pm 0.71^{\mathrm{a}}$ & 27.62 \\
${ }^{a}$ All the data are mean \pm SD. Different superscripted letters indicate significant difference within the same volume $(P<0.05)$. & 5.46
\end{tabular}

magnetic field direction, the $\Delta E$ gradually decreased. A smaller $\Delta E$ indicated a similarity in color quality between the treated sample and the fresh one. Under the uniform magnetic field, the ice crystal size was decreased by magnetic field non-thermal effect due to the molecular current in complex electrolyte system of the food, which helped maintain the relative integrity of the cells and prevented the release of color pigments. Polyphenol oxidase cannot fully come into contact with the pigments, causing an inhibition of enzymatic browning. In contrast, the large ice crystals formed under conventional freezing reduce the puree quality due to the increased contact area between polyphenol oxidase and the substrate without the magnetic field, thereby promoting enzymatic browning. Besides, the activity of endogenous enzymes, such as polyphenol oxidase and chlorophyllase, could be influenced by the magnetic field, which contributed to the preservation of natural color of the natural avocado puree..$^{27}$ Also, Gao et al. reported that alternating magnetic field-assisted-frozen the lotus root slices showed excellent color quality after thawing, due to the magnetic field $\left(50 \mathrm{~Hz}, 1.2 \mathrm{~A} \mathrm{~m}^{-1}\right)$, which inhibited the activity of polyphenol oxidase and delayed the browning of lotus root slices. ${ }^{28}$ However, Owada and Kurita found no significant changes in the color and odor of orange under freezing with or without applying the magnetic field $(100 \mathrm{Gs}){ }^{29}$

\subsection{Characteristic parameters}

The data presented in Fig. 4 reveals that the firmness of the frozen puree is lower than that of fresh sample due to water loss during the freezing process. The parameter of the control was also significantly lower than that in the groups subjected to magnetic field groups. With the increase of the dimension of the magnetic field, the hardness showed an increasing trend. As shown in Fig. 4, no significant differences in coating property between the magnetic field-assisted frozen sample and the control. All samples had the same spreadability level, which is a key textural attribute affecting consumers' acceptance. ${ }^{30}$ The viscosity of the groups subjected to magnetic field was higher than that of the control (Fig. 4). The improvement was observed when the direction of the magnetic field increased. The higher the viscosity of the sample, the smaller the free volume, indicating that a limited gap space was left for ice crystal growth when water molecules were crystallized in the sample. It would also inhibit the diffusion of other molecules and the recrystallization of water under 3D magnetic field.
In another study, magnetic fields played an active role in the maintenance of tissue structure as cucumber was frozen; the field changed cell membrane proteins, improving the efficiency of ions transport. ${ }^{14}$ Also, fresh meat products subjected to air- and cryo-freezing showed a differential firmness, suggesting that different freezing procedures were associated with the degree of cell membrane damage. However, the firmness of thawed meat was not significantly reduced compared with fresh meat that had previously been subjected to radio-frequency cryo-freezing. ${ }^{31}$

\subsection{Antioxidant capacity}

The antioxidant activity of the puree after the freezing decreased significantly. In particular, the DPPH-scavenging activity decreased from $69.18 \%$ to $56.22 \%$, and the FRAP value decreased from $18.5 \%$ to $14.75 \%$ (Fig. 5). Both the antioxidant activity of DPPH radical scavenging and FRAP of the frozen samples under the applied magnetic field was significantly higher than that of the control. Specifically, 3D magnetic field $\left(B_{X+Y+Z}\right)$ could maintain the antioxidant activity of avocado puree. Similarly, Zhao et al. compared the antioxidant activities of astaxanthin extracted from Haematococcus pluvialis subjected to various fields, including maceration, microwave, ultrasound, and magnetic field, and found that the products obtained upon

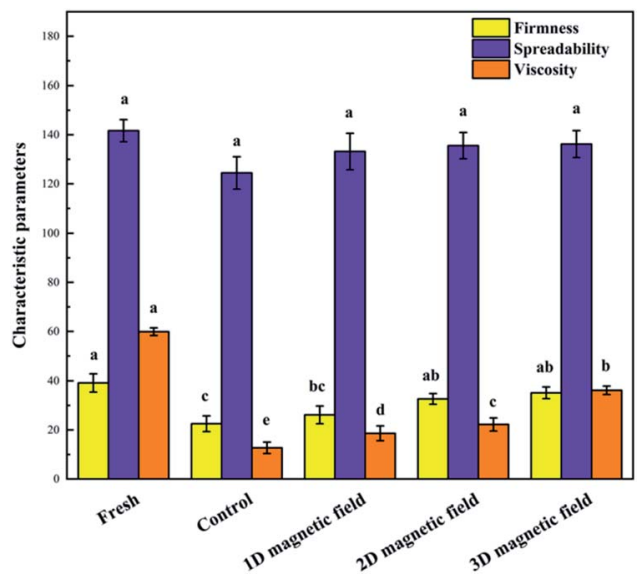

Fig. 4 Characteristic parameters of fresh avocado puree after the freezing with or without uniform magnetic field. Note: fresh, fresh avocado puree; control, freezing without the magnetic field; 1D magnetic field, freezing under $B_{X} ; 2 D$ magnetic field, freezing under $B_{X+Y} ; 3 D$ magnetic field, freezing under $B_{X+Y+Z}$. Different superscripted letters indicate significant difference within the same figure $(P<0.05)$. 

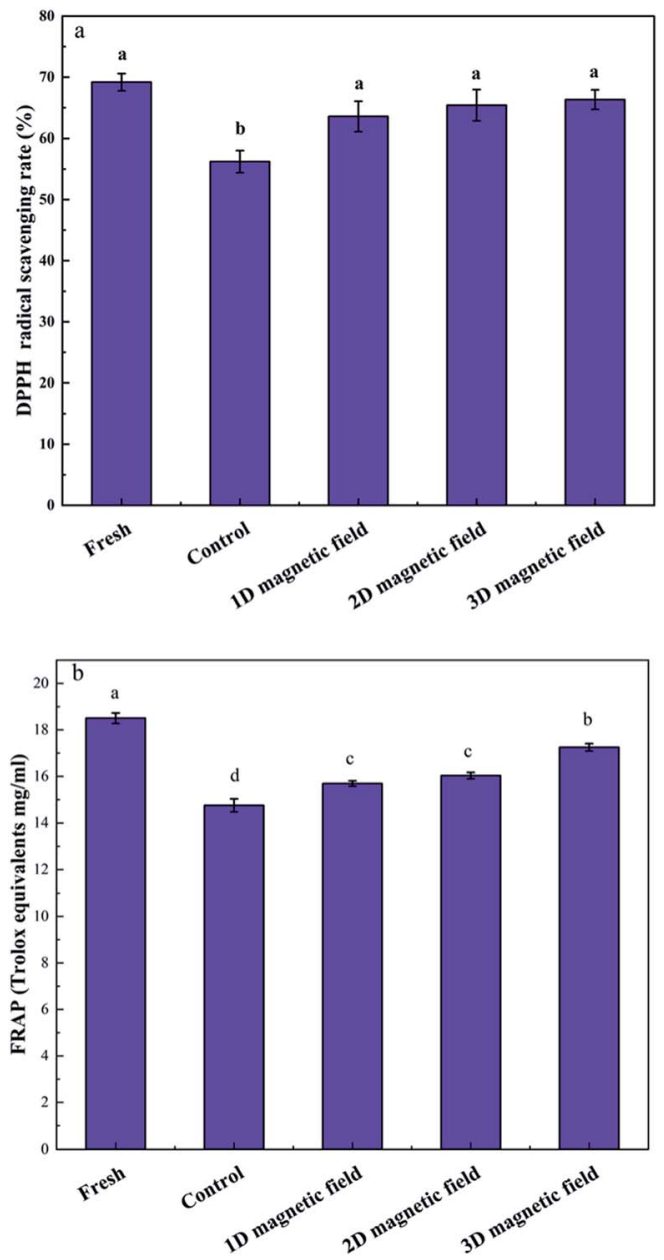

Fig. 5 Antioxidant activity of avocado puree after the freezing with or without uniform magnetic field. Note: fresh, fresh avocado puree; control, freezing without the magnetic field; 1D magnetic field, freezing under $B_{X} ; 2 D$ magnetic field, freezing under $B_{X+Y} ; 3 D$ magnetic field, freezing under $B_{X+Y+Z}$.

the magnetic field treatment showed the highest free radical inhibitory activity $\left(20.58 \%\right.$, at low concentration).$^{32}$

\subsection{Relative magnetic permeability}

The relative magnetic permeability of fresh avocado was 1.0089 , and that of the ferrous gluconate group was 1.0115 (Fig. 6). The parameter is crucial to characterize the magnetization of materials in an external magnetic field. It also represents the conduction of magnetic field within a material. The high magnetic permeability of the materials indicates a remarkable response under alternating magnetic fields. This demonstrated that the food material had extremely-low magnetic permittivity, even with the addition of $\mathrm{Fe}^{2+}$ ions. Furthermore, agricultural products or biological tissues generally exhibit low magnetic permeability so that there is no significant response under oscillating magnetic field. Accordingly, these samples theoretically have no hindrance on the magnetic field. Iron is a paramagnetic material, which exhibits excellent magnetic conduction. Therefore, when the avocado puree was added with

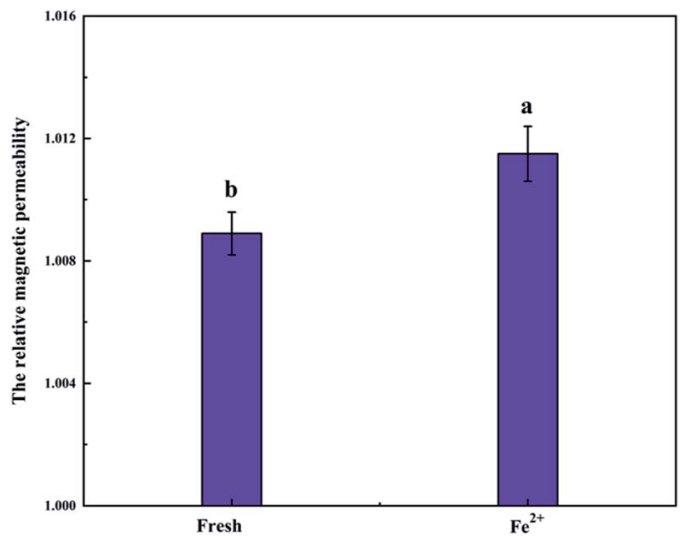

Fig. 6 The relative magnetic permeability of fresh avocado puree with or without ferrous gluconate.

ferrous gluconate, the magnetic permeability was slightly increased. Although no magnetic domain (or magnetite crystal) was formed in the avocado puree, remaining a non-magnetic sample, the weak molecular current because of the oscillating magnetic field would change the crystalline morphology of water molecules in avocado puree during freezing. The impact on freezing process and quality of the avocado puree is inevitable.
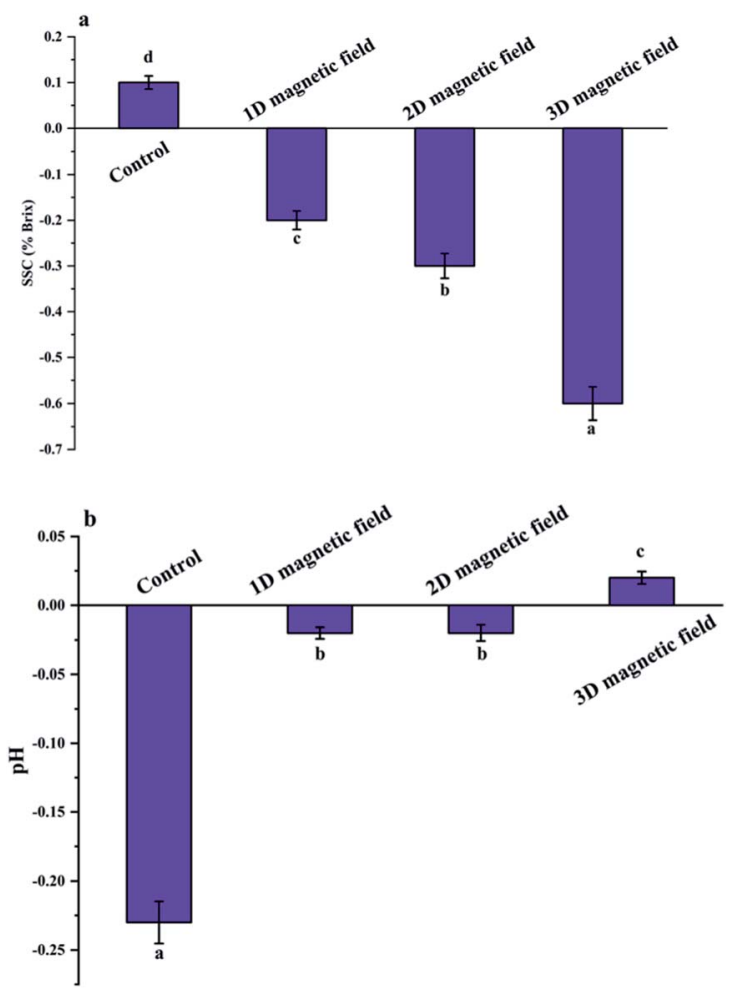

Fig. 7 SSC and $\mathrm{pH}$ value of avocado puree influenced by ferrous gluconate after freezing with or without uniform magnetic field (the comparison of the sample without Fe fortifier). Note: control, freezing avocado puree without the magnetic field; 1D magnetic field, freezing under $B_{X} ; 2 D$ magnetic field, freezing under $B_{X+Y} ; 3 D$ magnetic field, freezing under $B_{X+Y+Z}$. 
a
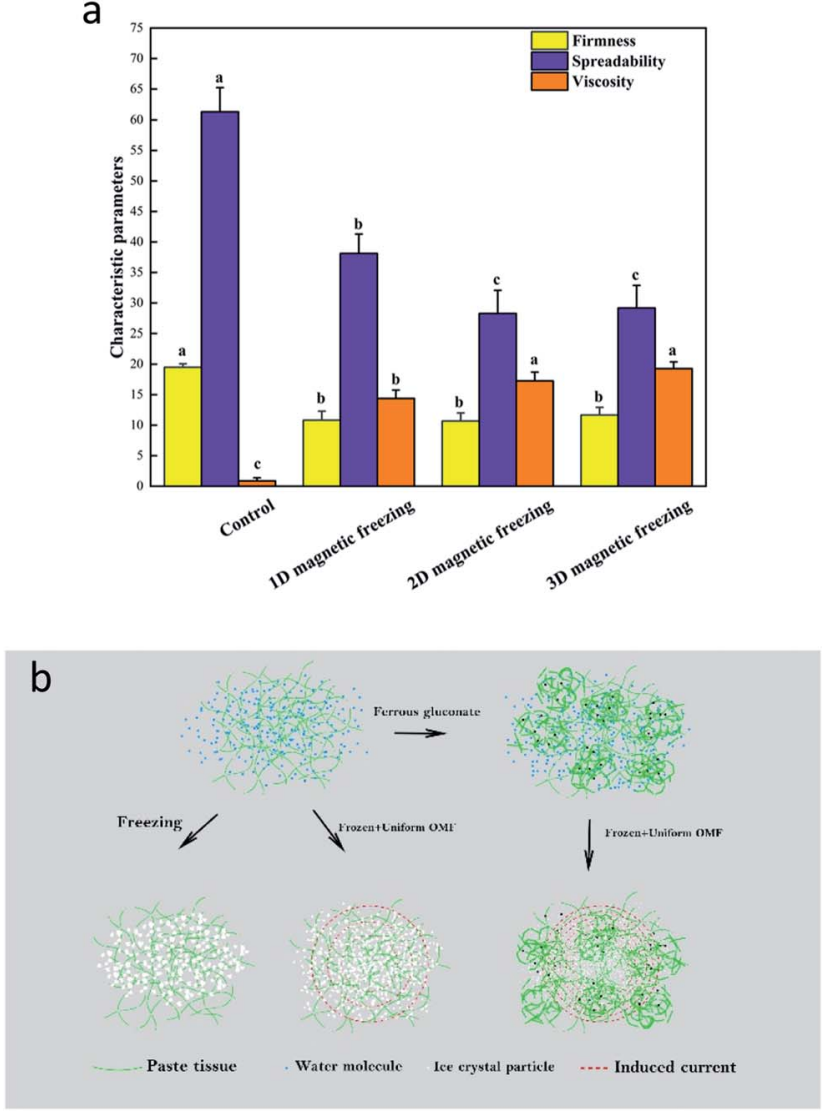

Fig. 8 (a) Characteristic parameters of avocado puree influenced by ferrous gluconate after the freezing with or without uniform magnetic field (the comparison of the sample without Fe fortifier); (b) the effect mechanism of oscillating magnetic field with high uniformity combined with ferrous ions on avocado puree during the freezing process. Note: control, freezing avocado puree without the magnetic field; 1D magnetic field, freezing under $B_{X} ; 2 D$ magnetic field, freezing under $B_{X+Y} ; 3 D$ magnetic field, freezing under $B_{X+Y+Z}$.

\subsection{Influence of Fe fortifier}

According to the data presented in Fig. 7, the SSC content in avocado puree added with ferrous gluconate is as follows (in descending order): the control, 1D magnetic field, 2D magnetic field, and 3D magnetic field. The $\mathrm{pH}$ value is as follows (in descending order): 3D magnetic field, 2D magnetic field, 1D magnetic field, and the control. These trends were consistent with that of the sample without the Fe fortifier. However, the difference was increased, suggesting that ferrous gluconate could be used to increase the response of avocado puree to the uniform magnetic field at $50 \mathrm{~Hz}$. After adding the $\mathrm{Fe}^{2+}$, the relative magnetic permeability of the sample was not significantly improved. But, when the alternating magnetic field was applied, based on the magnetoelectric effect, the small molecular current exerted a non-thermal effect on the freezing process.

The results shown in Fig. 8 reveal that, under the magnetic field, the Fe fortifier significantly affects the firmness, spreadability, and viscosity of avocado puree. The effect of the additive on avocado puree was mainly reflected in its spreadability,
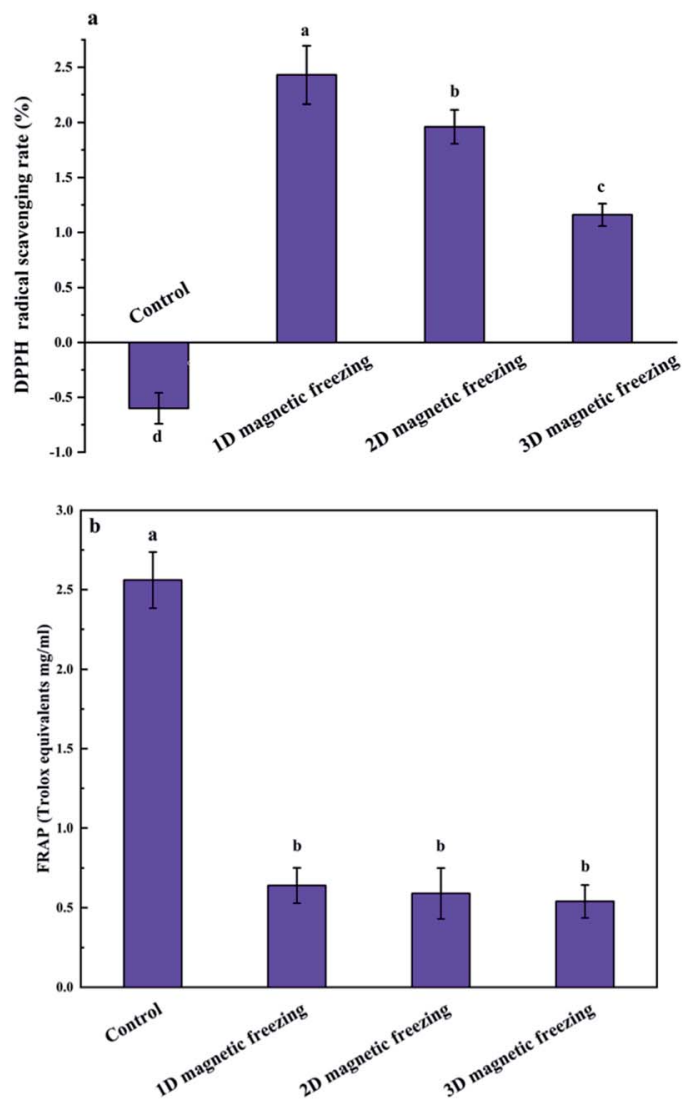

Fig. 9 DPPH radical scavenging activity and FRAP antioxidant activity of avocado puree influenced by ferrous gluconate after the freezing with or without uniform magnetic field (the comparison of the sample without Fe fortifier). Note: control, freezing avocado puree without the magnetic field; $1 D$ magnetic field, freezing under $B_{X} ; 2 D$ magnetic field, freezing under $B_{X+Y} ; 3 D$ magnetic field, freezing under $B_{X+Y+Z}$.

followed by viscosity and firmness. It suggested that ferrous ions bound to polysaccharides, formed metal complex structure and changed the freezing characteristics (Fig. 8b).

The data shown in Fig. 9 indicate that $\mathrm{Fe}^{2+}$ also significantly influence the DPPH radical scavenging activity and FRAP antioxidant activities after magnetic field-assisted freezing compared with the control. The antioxidant activity in the puree was maintained to a certain extent because of the Fe fortifier and the applied magnetic field. The interaction of the $\mathrm{Fe}^{2+}$ and the multi-direction magnetic field could exert a stimulating effect on avocado puree. The mechanical coupling of the $\mathrm{Fe}^{2+}$ to the cytoplasm is important for transducing the magnetism in tissue. In addition, Kobayashi and Kirschvink suggested that the magnetically induced oscillation of metal ions provides a plausible mechanism for the disruption of ice-crystal nucleation in supercooled water. ${ }^{33}$

\section{Discussion}

This study focused on the effects of a highly-uniform magnetic field on the quality of freeze-thawed avocado puree. During the process, a magnetic field of $4 \mathrm{mT}$ was applied on the sample in 
different directions (1D, 2D, 3D). Most food ingredients are electrically conductive, thus, the space electric field induced by alternating magnetic field continues producing circular molecular currents in the sample. ${ }^{34}$ During the freezing, the application of the 3D magnetic field on avocado puree implied that a stable periodic molecular current impeded the growth of ice crystals. Previous studies on the frozen quality of food or biological organs often applied an oscillating magnetic field or a static magnetic field. ${ }^{35,36}$ Abedini concluded that an alternating magnetic field $(0.1 \mathrm{mT}, 60 \mathrm{~Hz})$ combined with an electric field, inhibited ice crystal formation through non-thermal mechanisms, which were helpful for cell and tissue cryopreservation. ${ }^{37}$

Other researches have demonstrated that the quality of foods was not significantly influenced by the magnetic field-assisted freezing. ${ }^{38,39}$ At higher temperature, Bourget concluded that magnetic field, at the low doses used in their experiments $(2.5$ $\mathrm{mT}$ ), did not influence the ripening of tomato fruit during storage at $16{ }^{\circ} \mathrm{C}$, and that a higher intensity of magnetic field may be necessary to have an impact on the ripening behavior of tomatoes. ${ }^{40}$ However, the available information on the uniformity of magnetic field during the freezing process is insufficient during the freezing. In this work, an oscillating magnetic field with high uniformity was applied in multiple directions for the freezing, and was found to induce an all-around weak molecular current inside the sample. Moreover, when Fe nutrient fortifier was coupled with the surrounding cytoplasm, the magnetic field non-thermal effect during freezing was enhanced.

\section{Conclusions}

Under oscillating magnetic fields with strength of $4 \mathrm{mT}$, frequency of $50 \mathrm{~Hz}$, and homogeneity degree of $98 \%$, besides the significant decrease of the freezing point of avocado puree, the temperature zone for maximum ice formation and phase transformation time were also reduced. The effect of the magnetic field on the physicochemical properties of freezethawed avocado puree was also considerable. While the loss of soluble organic acids in avocado puree was relatively alleviated by the magnetic field. Compared with the control, the sample subjected to the magnetic field had a higher $\mathrm{pH}$ value and soluble solid content, particularly with the 3D magnetic field. The color value the group subjected to the 3D field was similar to that of the fresh sample, which implied that enzymatic browning in the avocado puree during the freezing under the magnetic field was lower. The inductive eddy currents were considered to maintain the product quality during the freezing process, preventing the formation of large size ice crystals. It suggested that the uniform magnetic field caused inadequate contact between polyphenol oxidase and substrates, leading to reduced activity of chlorophyll enzymes, resulting in delayed chlorophyll degradation. Simultaneously, the antioxidant activity of the samples after 3D magnetic field-assisted freezing was similar to that of fresh samples. The study indicated that applying a uniform magnetic field in three-direction can contribute to maintaining the physicochemical properties of freeze-thawed avocado puree.

\section{Conflicts of interest}

There are no conflicts of interest.

\section{Abbreviation}

$\begin{array}{ll}\text { DPPH } & \text { 1,1-Diphenyl-2-picrylhydrazyl } \\ \text { FRAP } & \text { Ferric reducing antioxidant power } \\ \text { AOAC } & \text { Association of analytical communities } \\ \text { AC } & \text { Alternative current } \\ \text { DC } & \text { Direct current } \\ \text { SSC } & \text { Soluble solid content }\end{array}$

\section{Acknowledgements}

The study was financially supported by the National Key Research and Development Program of China (2017YFD-0400404), the Nature Science Foundation of Jiangsu Province (No. BK20170182, BK20180608), the Agricultural Science and Technology Independent Innovation Funds of Jiangsu Province (CX(19)3069, CX(19)3367), and Open Fund of Beijing Key Laboratory for Food Non-Thermal Processing (NTKF2018001) and the Opening Foundation of Jiangsu Key Laboratory of Advanced Food Manufacturing Equipment and Technology (No. FM-2018-05, FM-2019-05).

\section{References}

1 M. L. Dreher and A. J. Davenport, Food Sci. Nutr., 2013, 53, 738-750.

2 M. Dalvi-Isfahan, N. Hamdami and A. Le-Bain, Innovative Food Sci. Emerging Technol., 2016, 42, 151-156.

3 A. Le Bail, M. Orlowska and M. Havet, Handbook of Frozen Food Processing and Packaging, 2012, pp. 685-691.

4 C. James, G. Purnell and S. J. James, Food Bioprocess Technol., 2015, 8, 1616-1634.

5 E. Xanthakis, A. Le-Bail and H. Ramaswamy, Innovative Food Sci. Emerging Technol., 2014, 26, 176-181.

6 X. Zhan, Z. Zhu and D. Sun, LWT-Food Sci. Technol., 2019, 103, 212-221.

7 H. Kiani, Z. Zhang and A. Delgado, Food Res. Int., 2011, 44, 2915-2921.

8 Q. Sun, X. Zhao and C. Zhang, LWT-Food Sci. Technol., 2019, 108, 106-112.

9 J. H. Mok, J. Y. Her and T. Kang, J. Food Eng., 2017, 196, 2735.

10 M. Dalvi-Isfahan, N. Hamdami and A. Le-Bain, Innovative Food Sci. Emerging Technol., 2016, 37, 68-73.

$11 \mathrm{~W}$. Sun, $\mathrm{X} . \mathrm{Xu}$ and $\mathrm{W}$. Sun, in Proceedings of the IEEE International Conference on Properties and Applications of Dielectric Materials, 2007, pp. 774-777.

12 J. H. Mok, W. Choi and S. H. Park, Int. J. Refrig., 2014, 50, 137-145.

13 X. Cheng, M. Zhang and B. Adhikari, Int. J. Refrig., 2014, 44, 49-55. 
14 S. Zhao, Z. Yang and L. Zhang, J. Food Eng., 2018, 217, 24-33. 15 L. Otero, M. Perez-Mateos and A. C. Rodríguez, J. Food Eng., 2017, 200, 87-94.

16 A. E. Lysionek, M. B. Zubillaga, M. J. Salgueiro, R. A. Caro, M. Segal, N. Shafran, N. Shapira and J. R. Boccio, J. Nutr. Sci. Vitaminol., 2002, 48, 315-317.

17 AOAC, Official Methods of Analysis of the Association of Official Analytical Chemists, Association of Official Analytical Chemists, Arlington, VA, 17th edn, 2000.

18 X. Wei, L. Yin and M. Zhong, Food Sci., 2014, 35, 317-322.

19 X. Cao, X. Bi, W. Huang and J. Wu, Innovative Food Sci. Emerging Technol., 2012, 16, 181-190.

20 L. Otero and P. D. San, J. Food Eng., 2006, 72, 54-363.

21 M. Iwasaka, M. Onishi and S. Kurita, J. Appl. Phys., 2011, 109, $07 \mathrm{E} 320$.

22 H. Ino, Y. Suzuki and T. Katamura, Food freezer and food thawing apparatus, JP Pat., 291525, 2005.

23 Y. Lou, Research progress in the effect of magnetic field on the freezing process, Doctoral thesis, Shandong University, 2014.

24 M. Gao and C. Wang, Food Res. Dev., 2010, 31, 155-158.

25 S. K. Ku, J. Y. Jeong and J. D. Park, Korean J. Food Sci. An., 2014, 34, 597-603.

26 Y. B. Kim, J. Y. Jeong and S. K. Ku, Korean J. Food Sci. An., 2013, 33, 723-729.
27 K. Samak, U. Yoshiyuki and A. Sukanya, Postharvest Biol. Technol., 2011, 61, 124-130.

28 M. Gao, C. Zhang and G. Wu, Food Sci., 2008, 29, 322-324.

29 N. Owada and S. Kurita, US Pat. 6250087 B1, 2001.

30 L. Jozef and D. Kokini \& Ann, J. Texture Stud., 1982, 13, 211227.

31 A. Monica, M. Lara and P. Agnese, Food Res. Int., 2012, 46, 50-54.

32 Z. Zhao, X. Zhang and L. Fu, Food Bioprod. Process., 2016, 99, 197-203.

33 A. Kobayashi and J. L. Kirschvink, Cryobiology, 2014, 68, 163165.

34 S. Sanjay, K. S. Sudhir and K. Lynn, J. Food Eng., 2008, 87, 351-356.

35 Z. Zhou, H. Zhao and J. Han, CIESC J., 2012, 63, 1405-1408.

36 Y. Mochimaru, N. Kuji and M. Yamada, Hum. Reprod., 2008, 23, 146.

37 S. Abedini, M. Kaku and T. Kawata, Cryobiology, 2011, 62, 181-187.

38 T. Suzuki, Y. Takeuchi and K. Masuda, Trans. Jpn. Soc. Refrig. Air Cond. Eng., 2009, 26, 371-386.

39 M. Watanabe, N. Kanesaka and K. Masuda, Proceedings of the $23^{\text {rd }}$ IIR International Congress of Refrigeration: refrigeration for sustainable development, 2011, pp. 21-26.

40 S. Bourget, R. Corcuff and P. Angers, Acta Hortic., 2012, 945(945), 129-133. 